\title{
Pesquisa-Ação no Desenvolvimento de um Instrumento para Consulta de Enfermagem na Saúde da Mulher
}

\section{Denise Antunes de Azambuja Zocche ${ }^{1}$, Ana Paula da Rosa² e Elisangela Argenta Zanatta ${ }^{3}$}

\footnotetext{
${ }_{1}^{1}$ Departamento de Enfermagem da Universidade do Estado de Santa Catarina/UDESC, Brasil | denise.zocche@udesc.br | https://orcid.org/0000-0003-4754-8439

${ }^{2}$ Secretaria Municipal de Saúde de Chapecó, Santa Catarina, Brasil | ana.lopessrosa@gmail.com | https://orcid.org/0000-0002-1923-0899

${ }^{3}$ Departamento de Enfermagem da Universidade do Estado de Santa Catarina/UDESC, Brasil | elisangela.zanatta@udesc.br | https://orcid.org/0000-0002-7426-6472
}

Resumo: A consulta de enfermagem na assistência à saúde da mulher, constitui-se em uma estratégia potente para a efetivação da promoção da saúde dessa população. A implantação de um instrumento para consulta de enfermagem à mulher, construído coletivamente por enfermeiros e com base em evidências científicas, torna-se uma estratégia de conduta coerente com as necessidades de saúde das mulheres. Objetivo: construir um instrumento para consulta de enfermagem à saúde da mulher, com base na Classificação Internacional para as Práticas em Enfermagem. Método: estudo qualitativo, do tipo pesquisa-ação, aplicada no âmbito da Atenção Primária em Saúde (APS), em cinco etapas, adaptadas do método proposta por Thiollent: diagnóstico situacional; coleta de dados; seminários; construção do instrumento; e publicização dos resultados. Os dados foram coletados por meio de entrevistas e quatro seminários, com 10 enfermeiras, que realizavam consulta de enfermagem a mulheres na Atenção Primária à saúde. Os dados foram analisados, segundo Bardin, e discutidos à luz das recomendações científicas nacionais e internacionais para a promoção da saúde da mulher. Os diagnósticos de enfermagem, as intervenções e os resultados esperados foram elaborados com base na Classificação Internacional para a Prática de Enfermagem. À medida que os campos do instrumento foram sendo elaborados e discutidos nos seminários, as mudanças propostas pelo grupo já eram tratadas para serem incorporadas na etapa de discussão dos seminários seguintes. Resultado: $O$ instrumento apresenta os seguintes campos: identificação; histórico, diagnósticos e intervenções de enfermagem; e resultados esperados para os motivos mais comuns de procura das mulheres por atendimento na APS. Foram elencados 10 diagnósticos, 28 intervenções de enfermagem e 10 resultados esperados a partir da Classificação Internacional para a Prática de Enfermagem. Conclusões: o método de pesquisa-ação permitiu a interação, reflexão e a construção de um instrumento para subsidiar a consulta de enfermagem para a saúde da mulher.

Palavras-chave: Consulta de Enfermagem; Atenção Primária a Saúde; Saúde da Mulher; Pesquisa-Ação; Pesquisa Aplicada.

\section{Research-Action in the Development of Women's Health Nursing Consultation}

Abstract: Nursing consultation in women's health care is a powerful strategy for effectively promoting the health of this population. The implantation of an instrument for nursing consultation specifically for women, built collectively by nurses and based on scientific evidence, becomes a strategy consistent with the health needs of women. Objective: to build an instrument for nursing consultation on women's health, based on the International Classification for Nursing Practices. Method: qualitative, research-action type study, applied within the scope of Primary Health Care, in five stages adapted from the method proposed by Thiollent: situational diagnosis, data collection, seminars, construction of the instrument and publication of the results. collected through interviews and four seminars, with 10 nurses, who performed nursing consultation to women in Primary Health Care. The data were analyzed according to Bardin and discussed in the light of national and international scientific recommendations for the promotion of women's health. Nursing diagnoses, interventions and expected results were prepared based on the International Classification for Nursing Practice. As the fields of the instrument were being elaborated and discussed in the seminars, the changes proposed by the group were already dealt with to be incorporated in the discussion stage of the following seminars. Result: The instrument has the following fields: identification; history, diagnoses and nursing interventions and expected results for the most common reasons for women seeking care in PHC. Ten diagnoses, twenty-eight nursing interventions and ten expected results from the International Classification for Nursing Practice were listed. Conclusions. The action-research method allowed interaction, reflection and the construction of an instrument to support nursing consultation for women's health.

Keywords: Nursing Consultation; Primary Healthcare; Women's Health; Research-Action; Applied Research. 


\section{Introdução}

A Atenção Primária a Saúde (APS) é a principal porta de entrada do usuário no sistema de saúde, e o enfermeiro, nesse cenário, exerce atividades técnicas, administrativas e educativas inerentes à sua profissão. Assim, o estabelecimento de vínculo entre o profissional e a mulher, tem por objetivo reduzir preconceitos ou tabus contra as mulheres e ofertar promoção à saúde e prevenção de agravos (Zocche et al., 2017). Segundo a Política Nacional de Atenção Básica, as atribuições do enfermeiro, incluem realização de grupos de educação em saúde, procedimentos, consulta de enfermagem, solicitação de exames complementares, prescrição de medicamentos e encaminhamentos para outros serviços. Essas atribuições devem ser realizadas desde que estejam regulamentas pelos órgãos de classe e previstas em protocolos (Brasil, 2017).

São muitos os desafios para implementar os princípios da APS, tendo em vista que o modelo assistencial preconizado prevê uma assistência centrada nas necessidades de saúde da população e, ainda, há pouca resolutividade na assistência de enfermagem na saúde da mulher, pois observa-se uma descontinuidade da terapêutica e limitação do atendimento (Pitilin \& Peloso, 2017).

Em um estudo realizado sobre a interação entre enfermeiras e usuárias do Sistema Único de Saúde (SUS) na APS, durante a consulta de enfermagem (CE), para realizar a coleta do exame citopatológico, ficou evidente que não há sensibilização das profissionais para demandas trazidas pelas usuárias ou que as soluções apresentadas pelas enfermeiras não são tomadas pelas usuárias, como satisfatórias (Meneghal et al, 2021).

Dessa forma, os protocolos assistenciais traduzem um respaldo técnico e conferem um caráter legal ao trabalho do enfermeiro, podendo conter de forma detalhada, a realização da consulta, histórico, diagnóstico, intervenção, resultados esperados e avaliação de enfermagem (Pereira \& Oliveira, 2018).

No contexto da realização da CE, o enfermeiro identifica situações do processo saúde/doença, levando em consideração meios científicos para este fim, objetivando a prescrição e implementação de enfermagem que possam contribuir para a prevenção, recuperação, reabilitação e promoção da saúde do indivíduo/família. Na realização da $\mathrm{CE}$, o enfermeiro tem papel essencial na criação de vínculos e na realização de atendimentos humanizados e qualificados. Para tanto, dispõe com a ferramenta de trabalho Processo de Enfermagem (PE), instituída pela Resolução no 358/2009, do Conselho Federal de Enfermagem (COFEN) (Conselho Federal de Enfermagem [COFEN], 2009).

Para que a CE se desenvolva de forma sistematizada, se faz necessário o cumprimento das seguintes etapas: coleta de dados de enfermagem (ou histórico de enfermagem); diagnóstico de enfermagem; planejamento de enfermagem; e implementação e avaliação de enfermagem (COFEN, 2009).

Com vistas a subsidiar as etapas do diagnóstico de enfermagem, no PE, é importante adotar um Sistema de Linguagem Padronizado (SLP) que ordena termos ou expressões que compõem os diagnósticos, intervenções, avaliações e resultados esperados componentes do PE. Entre eles, destaca-se a Classificação Internacional para a Prática de Enfermagem (CIPE $®)$, que é uma terminologia padronizada que representa o domínio da prática de enfermagem em âmbito mundial. É uma tecnologia de informação que proporciona a coleta, armazenamento, análise de dados de enfermagem em diversos cenários (Primo et al., 2018).

Diante do exposto e da dimensão da problemática apresentada e, ainda, considerando a necessidade da criação de um instrumento para embasar a consulta de enfermagem à saúde da mulher, o presente estudo objetivou construir um instrumento para consulta de enfermagem à saúde da mulher, com base na Classificação Internacional para as Práticas em Enfermagem. 
Esse estudo fez parte do trabalho de conclusão de curso apresentado ao Mestrado Profissional em Enfermagem na Atenção Primária à Saúde da Universidade do Estado de Santa Catarina e integra um macroprojeto de pesquisa intitulado "Estratégias para a implementação da Sistematização da Assistência de Enfermagem no cuidado à mulher e à criança", contemplado pelo Edital CAPES/COFEN n² 27/2016.

\section{Metodologia}

Trata-se de pesquisa qualitativa, método que contribui muito com estudos relacionados à atenção em saúde (Pope \& Mays, 2009), na modalidade pesquisa-ação. Thiollent (2011, p. 20) define a pesquisa-ação como "[...] um tipo de pesquisa social com base empírica que é realizada [...] em estreita associação com uma ação ou com a resolução de um problema coletivo e no qual os pesquisadores e os participantes [...] estão envolvidos de modo participativo".

A construção e validação do instrumento compreendeu cinco etapas: diagnóstico situacional; coleta de dados; seminários; construção do instrumento; e publicização dos resultados. Na primeira etapa, realizou-se uma revisão integrativa, com vistas a identificar, na literatura, como a CE na saúde da mulher está sendo desempenhada na APS, considerando as etapas do Processo de Enfermagem.

Em seguida, iniciou-se a coleta de dados, por meio de entrevistas com 10 enfermeiras e após foram realizados quatro grupos focais (GF) com sete enfermeiras das 10 entrevistados. Todos foram convidados a participar dos GF, somente sete aceitaram, os demais alegaram não conseguirem se afastar de suas atividades para participar. As entrevistas tinham por objetivo conhecer e avaliar a forma como a consulta de enfermagem à saúde da mulher era realizada, e, assim, subsidiar e elaboração dos roteiros de condução dos grupos focais.

O GF vai além de uma técnica de coleta de dados, sendo um importante mecanismo de intervenção, uma vez que viabiliza discussões e elaboração de estratégias para resolução de problemas comuns, pautados na experiência compartilhada pelos participantes (Sehnem et al., 2015). Essa técnica permitiu investigar e eleger quais os elementos que seriam incorporados ao instrumento de CE à saúde da mulher de forma coletiva.

Desenvolveu-se quatro GF, nos meses de julho de 2018 a abril de 2019, nas dependências da Universidade do Estado de Santa Catarina, organizada em formato de semicírculo, favorecendo o contato visual e a interação entre os participantes, o mediador e o relator, com o tempo médio de duração de cada GF de 3 horas e 30 minutos. Os áudios dos GF foram gravados e, transcritos, a fim de produzir material (conteúdo) a ser avaliado e validado nos encontros subsequentes. Cada grupo foi caracterizado pela letra (G), seguida do número que explicita a ordem de realização do grupo, a saber GF1, GF2, GF3, GF4.

No desenvolvimento dos seminários, os GFs foram organizados, a partir de roteiros préestabelecidos, de forma a abarcar todas as etapas do PE.

Os temas trabalhados nos GFs estão apresentados no Quadro 1.

Quadro 1. Temas desenvolvidos nos grupos focais.

\begin{tabular}{ll}
\hline \multicolumn{1}{c}{ Grupo Focal/Temas } & \multicolumn{1}{c}{ Temas } \\
\hline GF1 - "Resgatando & Devolutiva das entrevistas.Conceitos e \\
conceitos e pensando as & discussão sobre etapas da CE. \\
etapas da CE". & Levantamento dos três principais motivos \\
& para a procura de atendimentos. \\
GF2 - "Validando a primeira & Apresentação dos principais motivos de \\
etapa do roteiro da CE". & busca por atendimento à mulher, elencados \\
& pelo GF1. \\
& Construção dos diagnósticos a partir da \\
& CIPE®, para os principais motivos.
\end{tabular}




\begin{tabular}{ll}
\hline \multicolumn{1}{c}{ Grupo Focal/Temas } & \multicolumn{1}{c}{ Temas } \\
\hline GF3 - "Validando a segunda & Elaboração do planejamento e prescrição de \\
etapa da CE. & enfermagem. \\
& Escolhas dos diagnósticos de enfermagem, \\
& implementação e avaliação. \\
GF4 - "Validação do roteiro & $\begin{array}{l}\text { Apresentação e validação do roteiro de CE } \\
\text { da CE". }\end{array}$ \\
& $\begin{array}{l}\text { Aplicação do Índice de Validade de } \\
\text { Conteúdo (IVC). }\end{array}$ \\
\hline
\end{tabular}

O conteúdo das entrevistas e dos GFs, foi analisado conforme preceitos de Bardin, e à luz das recomendações científicas para a promoção da saúde da mulher e com base na CIPE®. (Bardin, 2016).

A exploração do material consistiu na codificação dos dados, com um recorte do material (palavras, frases, expressões), buscando elementos que se repetiam ou que possuíssem a mesma semântica. À medida que o instrumento era discutido e revisado, as mudanças propostas pelo grupo já eram analisadas e, novas questões foram sendo incorporadas nos seminários seguintes.

A última etapa foi a da publicização, que compreendeu apresentação ao Conselho Municipal de Saúde, do instrumento de CE a ser integrado ao protocolo municipal de enfermagem na saúde da mulher.

A pesquisa faz parte de um macroprojeto intitulado "Estratégias para a implementação da Sistematização da Assistência de Enfermagem no cuidado à mulher e à criança na perspectiva da Teoria Transcultural de Madeleine Leininger", o qual foi submetido ao Comitê de Ética em Pesquisa (CEP) da UDESC, CAAE no 79513617.6.0000.0118, via Plataforma Brasil e aprovado pelo Parecer № 2.630.923.

\section{Discussão}

A revisão integrativa de literatura, contribuiu na parte de construção dos roteiros para a realização dos seminários, sobre conceitos e etapas do PE. As entrevistas indicaram os três principais motivos de procura de atendimento pelas mulheres: sintomas urinários, corrimento vaginal e uso de métodos contraceptivos.

O motivo de procura pelo serviço, por sintomas urinários vai ao encontro do estudo de (Nerbass et.al., 2021 ), que indica dados sobre a prevalência de infecções do trato urinário, especialmente na população feminina, em gestantes e mulheres diabéticas.

O corrimento vaginal é uma queixa comum e prevalecendo na idade reprodutiva, e em serviços que atendem a infecções sexualmente transmissíveis, é o primeiro motivo de procura ( Brasil, 2018). As políticas públicas de saúde ofertada as mulheres, no Brasil, têm investido nos últimos anos, em programas de prevenção da gravidez, e infecções sexualmente transmissíveis, por meio da oferta de métodos contraceptivos, e testes rápidos para detecção de HIV e gravidez (Leal et. al., 2018).

Frente ao exposto, os motivos de procura pelos serviços neste estudo, relato pelas enfermeira confirma o que a literatura tem revelado sobre a atenção a saúde a mulher na APS e as ações ofertadas na APS brasileira.

Todas as enfermeiras indicaram conhecer o PE e que realizam a CE. No entanto, não executavam o PE em todas as suas etapas.

As participantes desse estudo apresentam limitações e dificuldades muito parecidas com a de outros enfermeiros em outras regiões do país, como confirma o estudo de (Oliveira et. al., 2019) ao revelar que a maioria dos profissionais de enfermagem do Brasil, reconhece a SAE e o PE, como elementos importantes para o seu trabalho, porém a aplicação efetiva de ambos na prática dos cuidados clínicos de enfermagem, ainda é um problema a ser superado.

Para (Gutierres et.al., 2018) o uso de um SLP ainda é uma limitação, a ser superada pelos profissionais na busca pela qualidade do cuidado em saúde e enfermagem realizado. 


\section{Resultados}

\subsection{Elaboração do Instrumento de CE à Saúde da Mulher na APS}

No primeiro GF, realizou-se um resgate dos conceitos sobre o PE e SAE, e discutiu-se as etapas da CE. A assistência de enfermagem à saúde da mulher foi amplamente debatida pelo grupo e o instrumento, previamente elaborado pela autora, foi apresentado aos participantes. O primeiro campo do instrumento de CE é constituído pela identificação, incluindo dados adicionais aos já agregados no cadastro individual da usuária no prontuário eletrônico, elencados pelos participantes como significativos para planejar a assistência.

O segundo campo abrange a etapa do histórico, que inclui dados relevantes para avaliação clínica da mulher, obtidos entrevista e exame físico, fatores de risco, ciclo menstrual, antecedentes obstétricos, planejamento familiar e rastreamento dos cânceres de colo uterino e mama. O último campo descreve sinais e sintomas relatados pela mulher, bem como o exame físico feito pelo profissional, com descrição do exame especular e de mamas.

No segundo GF, discutiu-se sobre a experiência do uso do instrumento, as dificuldades apresentadas e a proposição das adequações para melhoria da prática da CE na APS, entre elas, a realização dos diagnósticos de enfermagem durante a consulta e a necessidade de criar estratégias para o enfrentamento dos problemas organizacionais, como a necessidade de um curso sobre SAE e CE na atenção à saúde da mulher para os participantes.

No terceiro GF, realizou-se a elaboração do planejamento e prescrição de enfermagem, a partir das escolhas dos diagnósticos de enfermagem, feita sob consulta a CIPE®., sua implementação e avaliação. A construção de todas as etapas do PE, foi adequada às normas e rotinas presentes no protocolo municipal de saúde da mulher. Foram elencados 10 diagnósticos de enfermagem, propostas 57 intervenções de enfermagem e 10 resultados esperados.

\subsection{Avaliação do Instrumento}

O quarto GF avaliou o conteúdo do instrumento de CE para a saúde da mulher. Para tanto, utilizou-se a avaliação de especialistas sobre o conteúdo, constituindo uma abordagem qualitativa; e após uma quantitativa, pela utilização do IVC, que mede o grau de concordância entre os juízes, consistindo na aplicação de uma escala de pontuação de 1 a 4, denominada escala Likert. Para calcular o IVC, deve-se revisar ou anular os itens que receberem 1 ou 2. A soma das respostas 3 e 4 dos participantes especialistas deve ser dividida pelo número total de respostas. O índice aceitável deve ser no mínimo 0,80 e maior que 0,90 , preferência (Coluci et al., 2015).

Para aplicação do IVC, foi utilizado um instrumento com pontuação em escala Likert: $1=$ inadequado; 2 = parcialmente adequado; 3 = adequado; 4 = totalmente adequado. $\mathrm{O}$ IVC teve resultado de 1,0, demonstrando alto nível de concordância entre os juízes. Os resultados estão descritos na Tabela 1, a seguir. 
Tabela 1. Avaliação dos juízes, pela escala de Likert, quanto ao instrumento para consulta de enfermagem na saúde da mulher

\begin{tabular}{lcccccccccc}
\hline \multicolumn{1}{c}{ Itens } & P1 & P2 & P3 & P4 & P5 & P6 & P7 & A (\%) & TA (\%) & IVC \\
\hline $\begin{array}{l}\text { Histórico de } \\
\text { Enfermagem }\end{array}$ & 4 & $\mathbf{3}$ & $\mathbf{3}$ & 4 & 4 & $\mathbf{3}$ & 4 & 42,86 & 57,14 & 1,0 \\
$\begin{array}{l}\text { Diagnósticos de } \\
\text { enfermagem }\end{array}$ & 4 & 4 & 4 & 4 & 4 & 4 & 4 & - & 100 & 1,0 \\
$\begin{array}{l}\text { Planejamento de } \\
\text { Enfermagem }\end{array}$ & 4 & $\mathbf{3}$ & 4 & 4 & $\mathbf{3}$ & 4 & 4 & 28,57 & 71,43 & 1,0 \\
$\begin{array}{l}\text { Implementação de } \\
\text { Enfermagem }\end{array}$ & $\mathbf{3}$ & $\mathbf{3}$ & 4 & 4 & 4 & 4 & 4 & 28,57 & 71,43 & 1,0 \\
$\begin{array}{l}\text { Avaliação de } \\
\text { Enfermagem }\end{array}$ & $\mathbf{3}$ & $\mathbf{3}$ & 4 & 4 & $\mathbf{3}$ & 4 & 4 & 48,86 & 57,14 & 1,0 \\
\hline
\end{tabular}

Legenda: $\mathbf{P}=$ participante, $\%$ Adeq = Grau de concordância Adequado, \% TA_totalmente adequado = Grau de concordância Totalmente adequado, IVC= Índice de validade de conteúdo.

O instrumento para CE na saúde da mulher, após validação, foi apresentado à Secretaria de Saúde do Município, sendo incorporado ao protocolo de enfermagem na atenção à saúde da mulher.

\section{Conclusões}

Os grupos focais foram resolutivos, pois oportunizaram momentos focados de estudo, de reflexão e ação sobre o fazer profissional, ou seja, a consulta de enfermagem baseada na $\mathrm{CIPE} \Theta$, com vistas a qualificar o trabalho do enfermeiro e a atender as necessidades de saúde das mulheres na atenção primária do município.

A consulta de enfermagem e a CIPE® são estratégias potenciais para a valorização da profissão e consolidação da identidade profissional.

\section{Referências}

Bardin, L. (2016). Análise de conteúdo. São Paulo: Edições 70.

Brasil. (2017). Portaria no 2436 de 21 de setembro de 2017. Aprova a Política Nacional de Atenção Básica, estabelecendo a revisão de diretrizes para a organização da Atenção Básica, no âmbito do Sistema Único de Saúde (SUS). Brasília, 2017.

Brasil. (2018). Portaria MS/SCTIE no 42, de 5 de outubro de 2018. Aprova o Protocolo Clínico e Diretrizes Terapêuticas para Atenção Integral às Pessoas com Infecções Sexualmente Transmissíveis (IST), no âmbito do Sistema Único de Saúde (SUS).Brasília, 2018.

Coluci, M. Z. O., Alexandre, N. M. C., \& Milani, D. (2015). Construção de instrumentos de medida na área da saúde. Ciência \& Saúde Coletiva, 20(3), 925-936. https://dx.doi.org/10.1590/1413-81232015203.04332013.

Conselho Federal de Enfermagem (COFEN). (2009). Resolução COFEN-358/2009. Brasília, DF: COFEN.

Gutierres, L.S., Santos, J.L.G., Peite, C.C., Menegon, F.H.A., Sebold, L.F.\&, Erdmann, A.L.(2018). Good practices for patient safety in the operating room: nurses' recommendations. Ver. Bras. Enferm , 71 (Suppl 6) [Internet].pp. 2775-82.

Leal, M.D.C, Szwarcwald C.L., Almeida. P.V.B., Aquino, E.M.L., Barreto, M.L.,\& Barros F, Victora C.( 2018). Saúde reprodutiva, materna, neonatal e infantil nos 30 anos do Sistema Único de Saúde (SUS). Cien Saude Colet; 23(6). pp. 1915-1928. 
Meneghel, S. N., Andrade, D. N. P., \& Hesler, L. Z. (2021). Conversas invisíveis: assuntos falados, mas não ouvidos em consultas ginecológicas. Ciência \& Saúde Coletiva [online]. 26 (1), pp. 275-284

Nerbass, F.B. et al. (2021). Female nurses have a higher prevalence of urinary tract symptoms and infection than other occupations in dialysis units. Brazilian Journal of Nephrology [online].

Pereira, J. G., Oliveira, M. A. C.(2018). Autonomia da enfermeira na Atenção Primária: das práticas colaborativas à prática avançada. Acta paul. enferm., São Paulo, 31(6), 627-635, dez.

Pitilin, É. B., \& Pelloso, S. M. (2017). Primary care sensitive admissions in pregnant women: associated factors based on the prenatal care process. Texto \& Contexto - Enfermagem, 26(2), e06060015. Epub July 03.

Pope, E. C., \& Mays, S. N. (2009). Pesquisa Qualitativa na Atenção à Saúde. Porto Alegre: Artmed.

Oliveira, M. R. et al. (2019). Nursing care systematization: perceptions and knowledge of the Brazilian nursing. Revista Brasileira de Enfermagem 72 (6) [online]. pp. 1547-1553

Primo, C. C., Resende, F. Z., Garcia, T. R., Duran, E. C. M., \& Brandão, M. A. G. (2018). Subconjunto terminológico da CIPE $®$ para assistência à mulher e à criança em processo de amamentação. Rev. Gaúcha Enferm. [Internet]. 39: e2017-0010.

Sehnem, G. D. et al. (2015). Utilização do grupo focal como técnica de coleta de dados em pesquisas: relato de experiência. Cienc Cuid Saude, v. 14, n. 2, p. 1194 - 1200, abr.- jun. 2015.

Thiollent, M. (2011). Metodologia da pesquisa-Ação. São Paulo: Cortez.

Zocche, D. A. A., Vendruscolo, C., Adamy, E. K., Ribeiro, K. P., \& Oliveira, M. C. B. (2017) Percepções de enfermeiros acerca da integralidade da atenção à saúde feminina. Rev enferm UFPE on line., 11, 4758-4766. 